\title{
Evaluation of the Pharmacokinetic Interaction between Lesogaberan (AZD3355) and Esomeprazole in Healthy Subjects
}

\author{
Mohammad Niazi, ${ }^{1}$ Debra G. Silberg, ${ }^{2}$ Frank Miller, ${ }^{3}$ Magnus Ruth ${ }^{1}$ and Ann A. Holmberg ${ }^{1}$ \\ 1 AstraZeneca R\&D, Mölndal, Sweden \\ 2 AstraZeneca Pharmaceuticals LP, Wilmington, Delaware, USA \\ 3 AstraZeneca R\&D, Södertälje, Sweden
}

Abstract

Background: Transient lower esophageal sphincter relaxations (TLESRs) have been identified as a primary cause of reflux events in patients with gastroesophageal reflux disease (GERD). GABA $_{\mathrm{B}}$ receptor agonists such as lesogaberan (AZD3355) have been shown to inhibit TLESRs in healthy subjects and patients with GERD, and, therefore, offer a novel therapeutic add-on strategy to acid suppression for the management of GERD. As lesogaberan is being developed as an add-on treatment for the management of patients with GERD who have a partial response to proton pump inhibitor (PPI) therapy, it is important to rule out any clinically important pharmacokinetic drug-drug interaction between lesogaberan and PPIs.

Objective: To evaluate the effect of esomeprazole on the pharmacokinetics and safety of lesogaberan and vice versa.

Study Design: This was an open-label, randomized, three-way crossover study. The study was open to healthy adult male and female subjects. The study subjects received treatment with, in random order, lesogaberan $(150 \mathrm{mg}$ twice daily [dose interval 12 hours]), esomeprazole ( $40 \mathrm{mg}$ once daily), and a combination of both, during 7-day treatment periods.

Main Outcome: The presence or absence of pharmacokinetic interactions between lesogaberan and esomeprazole was assessed by measuring the steady-state area under the plasma concentration-time curves during the dosing interval $\left(\mathrm{AUC}_{\tau}\right)$ and the maximum observed plasma concentration $\left(\mathrm{C}_{\text {max }}\right)$ for lesogaberan and esomeprazole.

Results: Thirty male subjects (mean age 23.2 years, 97\% Caucasian) were randomized to treatment and 28 subjects completed the study (one subject was lost to follow-up, and one subject discontinued due to an adverse event). The $95 \%$ confidence intervals of the geometric mean ratios for $\mathrm{AUC}_{\tau}$ and $\mathrm{C}_{\max }$ of lesogaberan and esomeprazole administered alone and concomitantly 
were within the recognized boundaries of bioequivalence (0.8-1.25). No new safety concerns were raised during this study. The number of patients with adverse events during treatment with lesogaberan alone $(n=17)$ and concomitantly with esomeprazole $(n=18)$ were comparable but higher than with esomeprazole alone $(\mathrm{n}=10)$. Paresthesia (episodic, mild, and transient), pharyngitis, and flatulence were the most frequently reported adverse events. Conclusions: There was no observed pharmacokinetic interaction between lesogaberan and esomeprazole when concomitantly administered to healthy subjects, and concomitant therapy was well tolerated.

Trial registration number (clinicaltrials.gov): NCT00684190

\section{Background}

$\mathrm{GABA}_{\mathrm{B}}$ receptor agonists such as lesogaberan (AZD3355) are known to inhibit transient lower esophageal sphincter relaxations, ${ }^{[1]}$ a primary cause of reflux in patients with gastroesophageal reflux disease (GERD). ${ }^{[2]}$ Indeed, pharmacodynamic studies suggest possible therapeutic utility for lesogaberan in patients with persistent GERD symptoms despite proton pump inhibitor (PPI) therapy. ${ }^{[3,4]}$

However, as lesogaberan was developed to be used as an add-on medication for the treatment of patients with a partial response to PPIs, it is important to establish the lack of any clinically important pharmacokinetic interaction between these agents. Therefore, the aim of the present study was to assess the steady-state pharmacokinetic interaction between lesogaberan and esomeprazole when administered alone and concomitantly to healthy subjects. Esomeprazole was chosen to represent the PPIs as a class because it is widely prescribed and mainly metabolized by cytochrome P450 (CYP) $2 \mathrm{C} 19$ and CYP3A4, ${ }^{[5]}$ as with omeprazole, ${ }^{[6,7]}$ lansoprazole, ${ }^{[8,9]}$ pantoprazole, ${ }^{[6,10]}$ and rabeprazole..$^{[1,12]}$

\section{Methods}

This open-label, randomized, three-way crossover study ${ }^{[13]}$ was conducted at a single Swedish center, in accordance with ethical principles and standards described in the Declaration of Helsinki and the International Conference on Harmonisation $(\mathrm{ICH}) /$ Good Clinical Practice (GCP) guidelines. Written informed consent was obtained from all participating subjects prior to the commencement of the study.

\section{Study Aims}

The aims of this study were to assess the pharmacokinetic interaction between lesogaberan and esomeprazole at steady-state and vice versa in healthy subjects via the assessment of the area under the plasma concentration-time curve (AUC) during the dosing interval $\left(\mathrm{AUC}_{\tau}\right)[12$ hours for lesogaberan and 24 hours for esomeprazole] and the maximum observed plasma concentration $\left(\mathrm{C}_{\max }\right)$ for lesogaberan and esomeprazole when administered alone and concomitantly. Additional study evaluations included the assessment of AUC from time zero until the last quantifiable concentration $\left(\mathrm{AUC}_{\mathrm{t}}\right)$, time to reach maximal plasma concentration $\left(t_{\max }\right)$, terminal elimination halflife $\left(t_{1 / 2}\right)$, and oral clearance $(\mathrm{CL} / \mathrm{F})$. The potential for lesogaberan to induce CYP3A4 was measured by the analysis of plasma $4-\beta$-hydroxy-cholesterol (4- $\beta \mathrm{OH}$-cholesterol) concentrations. The safety and tolerability of lesogaberan administered alone and concomitantly with esomeprazole were also assessed.

\section{Study Population}

Healthy male or female subjects aged 18-45 years with a body mass index of $19-30 \mathrm{~kg} / \mathrm{m}^{2}$ and a body mass of $50-100 \mathrm{~kg}$ were eligible for inclusion. Only surgically sterilized female subjects were included in the study, as the reproductive toxicology of lesogaberan is currently unknown. 
In order to ensure safety, subject exclusion criteria included (i) a clinically significant illness within 2 weeks prior to the first dose of study medication(s); (ii) a history of allergy/hypersensitivity, clinically significant orthostatic reactions/syncope, or of cardiovascular, respiratory, renal, hepatic, neurologic, mental, or gastrointestinal disease; (iii) ongoing allergy/hypersensitivity; or (iv) any concomitant condition/medication that could potentially modify the pharmacokinetics of the study drug(s).

\section{Study Design and Treatments}

The study flow is presented in figure 1. Following the pre-study screening visit, the study consisted of three 7-day treatment periods separated by washout periods of at least 13 days. On day 1 of the first treatment period, subjects were block randomized to random-order treatment with the following three treatment regimens: (i) hardcapsule modified-release formulation lesogaberan (150 $\mathrm{mg}$ twice daily [dosing interval 12 hours]) dosed on the morning and afternoon of days 1-6 and once on the morning of day 7; (ii) esomeprazole capsules ( $40 \mathrm{mg}$ once daily; formulated as esomeprazole magnesium trihydrate $44.5 \mathrm{mg}$ ) dosed on the morning of days $1-7$; or (iii) the two regimens combined and dosed as described.

Study medications were administered with a standardized volume $(240 \mathrm{~mL})$ of water to fasted subjects. Food was restricted from $11 \mathrm{pm}$ on the evening prior to administration of study medication until 1 hour post-morning dose on days 1-6 or until 4 hours after the morning dose on day 7; fluids were restricted from 1 hour pre-dose until 1 hour post-morning dose on days $1-6$ or until 2 hours after the morning dose on day 7 .
Standardized meals were provided 1 hour after morning dosing on days 1-6 and 4 hours after dosing on day 7. All study subjects were required to stay at the study center overnight on day 6 .

Additional study procedures included (i) daily monitoring of adverse events via self-reporting and direct questioning; (ii) orthostatic testing after the standardized breakfast on day 1, pre-dose on days 2 and 4, and 3 hours post-dose on day 7; and (iii) a physical examination, supine blood pressure and pulse measurements, and electrocardiography at the follow-up visit.

\section{Sample Collection and Analysis}

Plasma concentration-time profiles for lesogaberan and esomeprazole on day 7 were generated from blood samples $(2 \mathrm{~mL})$ collected from pre-dose until 12 hours post-dose: $0.5,1.0,1.5$, $2.0,2.5,3.0,3.5,4.0,5.0,6.0,8.0,10$, and 12 hours post-dose. Additional blood samples were taken for the determination of pre-dose lesogaberan plasma concentrations on days 4,5 , and 6 of the relevant treatment periods. In order to evaluate the potential for CYP3A4 induction, blood samples were also collected pre-dose on days 1 and 7 for the determination of $4-\beta \mathrm{OH}$-cholesterol plasma concentrations in subjects receiving lesogaberan alone.

Plasma samples were prepared by centrifugation (relative centrifugal force, $1500 \mathrm{~g}$ ) and immediately frozen $\left(-20^{\circ} \mathrm{C}\right.$ for lesogaberan and esomeprazole; $-70^{\circ} \mathrm{C}$ for $4-\beta \mathrm{OH}$-cholesterol). Samples were analyzed by an accredited laboratory (PRA International, Assen, the Netherlands) using reversephase liquid chromatography and mass spectrometry methods for lesogaberan and $4-\beta \mathrm{OH}$-cholesterol, and normal phase liquid chromatography with

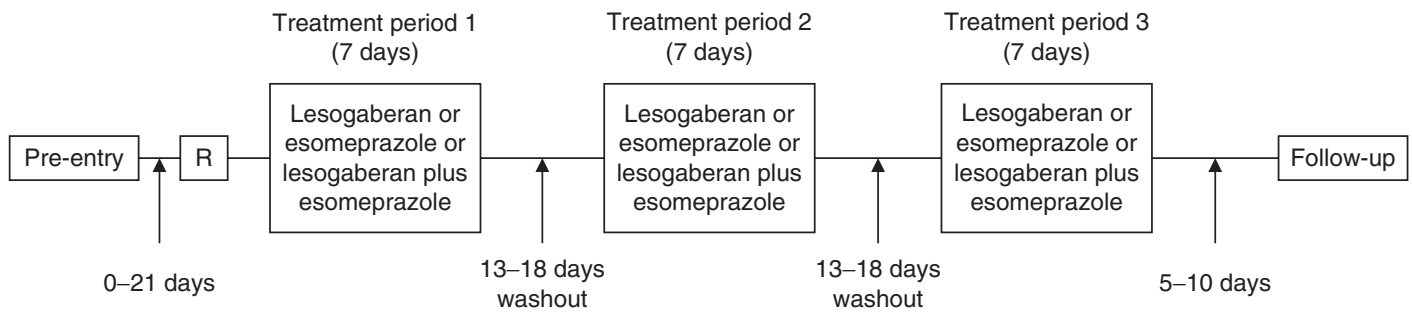

Fig. 1. Study design. $\mathbf{R}=$ randomization. 
ultra violet detection for esomeprazole. The lower limits of quantification for lesogaberan and esomeprazole were $0.03 \mu \mathrm{mol} / \mathrm{L}$ and $0.025 \mu \mathrm{mol} / \mathrm{L}^{[14]}$

\section{Statistical Analysis}

A total of at least 30 subjects were randomized to treatment to ensure at least 24 evaluable subjects at the end of the study. The calculations to determine the sample size were based on data from previous studies. ${ }^{[14]}$ For lesogaberan, it was assumed that the within-subject standard deviation (SD) of $\ln$ AUC and $\ln \mathrm{C}_{\max }$ would be approximately 0.2 , and that the within-subject $\mathrm{SD}$ of the difference in $\ln \mathrm{AUC}$ and $\ln \mathrm{C}_{\max }$ would then be approximately 0.28 . With 24 evaluable subjects, the probability that a $90 \%$ confidence interval (CI) of the ratio would be no wider than $0.86,1.16$ was $80 \%$. For esomeprazole, the expected within-subject SD of $\ln \mathrm{AUC}$ and $\ln \mathrm{C}_{\max }$ was approximately 0.22 and 0.32 . With 24 evaluable subjects, there was an $80 \%$ probability that the $90 \%$ CIs of the geometric mean ratio for AUC would be no wider than $0.85,1.18$, and that the geometric mean ratio for $\mathrm{C}_{\max }$ would be no wider than $0.78,1.27$.

\section{Analysis Sets}

The pharmacokinetic analysis set included all subjects who received lesogaberan or esomeprazole either alone or concomitantly, and had evaluable pharmacokinetic data with no major protocol deviations. The safety population was defined as all subjects who received at least one dose of lesogaberan or esomeprazole either alone or concomitantly, and for whom post-dose data were available.

\section{Methods of Statistical Analysis}

The pharmacokinetic analyses were performed using standard non-compartmental methods and WinNonlin Professional 4.1.b (or later) software by Phase I Services, Quintiles AB, Uppsala, Sweden.

The log-transformed variables $\mathrm{AUC}_{\tau}$ and $\mathrm{C}_{\max }$ were analysed using a mixed model ANOVA with fixed effects for sequence, period, and treatment, and a random effect for subject within sequence. The evaluation of the effect of lesogaberan on the pharmacokinetic variables of esomeprazole and vice versa were performed by calculating $90 \%$ and 95\% symmetrical $t$-distribution CIs for the mean differences in $\log \mathrm{AUC}_{\tau}, \log \mathrm{AUC}_{\mathrm{t}}$, and $\log \mathrm{C}_{\text {max }}$ when lesogaberan and esomeprazole were administered concomitantly versus alone. The CIs were antilog transformed to generate CIs for the ratios of geometric means and coefficients of variation. Here, we report only the wider 95\% CIs. A similar transformation to calculate the ratio of geometric means and corresponding 95\% CIs of 4- $\beta \mathrm{OH}$-cholesterol plasma concentration following administration of lesogaberan alone was performed.

\section{Results}

Subjects

A total of 30 healthy male subjects were randomized to treatment according to the three-way crossover design (table I). The three treatment sequences were generally well balanced with respect to baseline characteristics. Twenty-eight subjects completed the study without protocol violation and were included in the pharmacokinetic analysis set; one subject was lost to follow-up and one subject was discontinued from treatment due to an adverse event (fever).

\section{Pharmacokinetic Variables}

The steady-state plasma concentration-time curves for lesogaberan and esomeprazole, administered alone and concomitantly, are presented in figures 2 and 3. Analysis of trough plasma concentrations indicated that steady-state plasma concentrations for lesogaberan were attained prior to

Table I. Subject baseline characteristics $(n=30)$

\begin{tabular}{lll}
\hline Characteristic & Value $^{\mathrm{a}}$ & Range \\
\hline Age $[\mathrm{y}]$ & $23.2 \pm 3.9$ & $18-35$ \\
Height $[\mathrm{cm}]$ & $180.4 \pm 6.9$ & $166-195$ \\
Weight $[\mathrm{kg}]$ & $75.4 \pm 8.7$ & $59-93$ \\
Body mass index $\left[\mathrm{kg} / \mathrm{m}^{2}\right]$ & $23.3 \pm 2.7$ & $20-28$ \\
Ethnicity & & \\
$\quad$ White [n (\%)] & $29(97)$ & \\
$\quad$ Black or African American [n (\%)] & $1(3)$ & \\
\hline a Values represented as mean $\pm \mathrm{SD}$, unless stated otherwise.
\end{tabular}




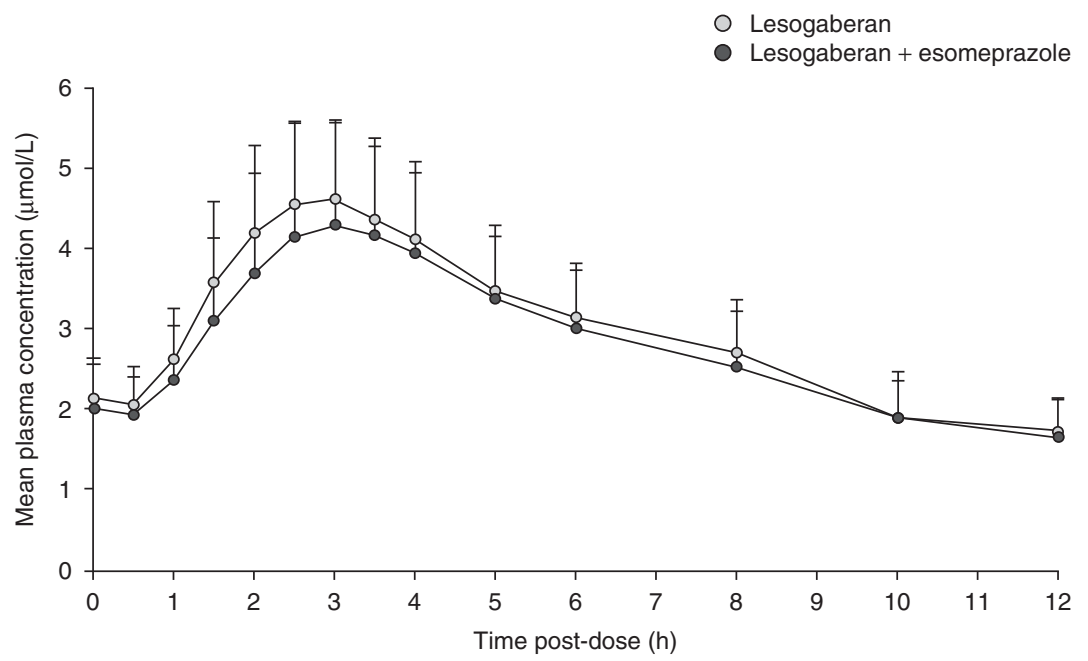

Fig. 2. Mean steady-state plasma concentration-time curves of lesogaberan when administered alone $(n=29)$ and concomitantly with esomeprazole $(n=28)$. Data are the combined arithmetic mean values SD of the two following treatment groups: (i) lesogaberan $150 \mathrm{mg}$ twice daily (bid) [dosed in the morning and afternoon] on days 1-6 and $150 \mathrm{mg}$ once daily (od) [dosed in the morning] on day 7; and (ii) lesogaberan $150 \mathrm{mg}$ bid on days $1-6$ and $150 \mathrm{mg}$ od on day 7 , administered concomitantly with esomeprazole $40 \mathrm{mg}$ od (dosed in the morning) on days $1-7$. Data are from day 7.

day 7. Although lag times were not recorded in this study, visual inspection of the plasma concentration-time profile shows that the lag times of lesogaberan and esomeprazole were not affected when dosed together, relative to dosing alone.

The $95 \%$ CIs of the geometric mean ratios for the $\mathrm{AUC}_{\tau}, \mathrm{C}_{\max }$, and $\mathrm{AUC}_{\mathrm{t}}$ of lesogaberan after concomitant administration with esomeprazole versus lesogaberan alone were contained within the accepted interval of bioequivalence (0.8-1.25, table II). The corresponding variables for esomeprazole, administered concomitantly with lesogaberan versus esomeprazole alone, were also contained within the accepted limits of bioequivalence (table II).

The geometric mean values of $\mathrm{C}_{\max }, \mathrm{t}_{1 / 2}$, and $\mathrm{CL} / \mathrm{F}$ of lesogaberan and esomeprazole were comparable when the drugs were administered alone or concomitantly. The corresponding median $t_{\max }$ values for lesogaberan were comparable, although the median $\mathrm{t}_{\max }$ of esomeprazole when administered concomitantly with lesogaberan was slightly shorter compared with esomeprazole alone (table III). The mean and ranges of $t_{\max }$ were comparable following administration alone (2.2 hours; range $1-4$ hours) and con- comitantly with lesogaberan (1.9 hours; range 1-4 hours).

During treatment with lesogaberan alone, the geometric mean concentration of 4- $\beta \mathrm{OH}$-cholesterol was slightly reduced from pre-dose on day 1 $(48.4 \mathrm{nmol} / \mathrm{L})$ to pre-dose on day $7(44.0 \mathrm{nmol} / \mathrm{L})$; the geometric mean ratio was 0.91 (95\% CI 0.88, 0.94).

\section{Safety and Tolerability}

There were no deaths or serious adverse events during the course of the study. One subject was discontinued from treatment while receiving lesogaberan concomitantly with esomeprazole due to fever that was considered to be related to a viral infection.

A total of 119 adverse events were reported by 27 of the 30 subjects throughout the study. The majority were either of mild or moderate intensity (106 and 10 events). Three adverse events that were graded as severe were reported (vasovagal syncope, forearm injury [bicycle accident], and myalgia). The event coded as vasovagal syncope without fainting occurred during a blood draw while the subject was pre-dose in day 7 of the esomeprazole alone treatment period. The number of subjects with adverse events during 
O Esomeprazole

- Esomeprazole + lesogaberan

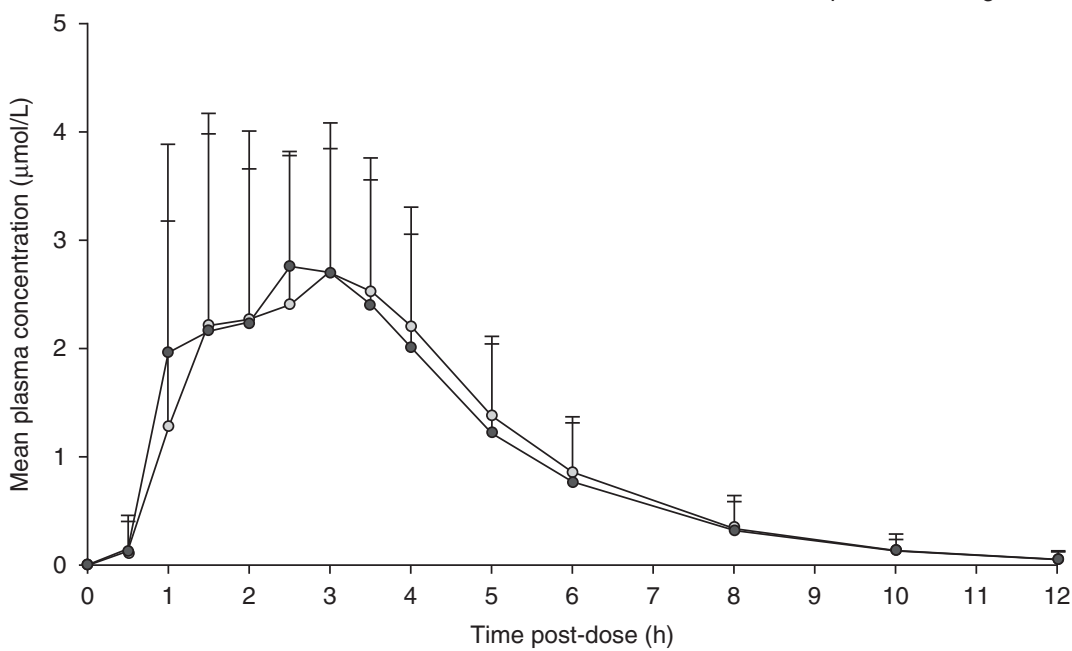

Fig. 3. Mean steady-state plasma concentration-time curves of esomeprazole, alone $(n=29)$ and concomitantly with lesogaberan ( $n=28)$. Data are the combined arithmetic mean values + SD of the two following treatment groups: (i) esomeprazole $40 \mathrm{mg}$ once daily (od) [dosed in the morning] on days 1-7; and (ii) lesogaberan $150 \mathrm{mg}$ twice daily (dosed in the morning and afternoon) on days 1-6 and $150 \mathrm{mg}$ od (dosed in the morning) on day 7 , administered concomitantly with esomeprazole $40 \mathrm{mg}$ od in the morning on days $1-7$. Data are from day 7.

treatment with lesogaberan alone $(n=17)$ was comparable to that during combined administration with esomeprazole $(\mathrm{n}=18)$, but higher than with esomeprazole alone $(n=10)$. The events that were reported by the greatest number of subjects during active treatment were transient paresthesia, pharyngitis, and flatulence (table IV). In all subjects with paresthesia $(n=5)$, episodes were mild in severity.

On day 1 in subjects treated with lesogaberan alone, orthostatic testing showed that the mean increase in reflectory pulse from a supine to standing position was higher at 1 hour post-dose (increase in beats per minute [bpm] measured after 1 and 3 minutes of standing, 24.7 and $27.0 \mathrm{bpm})$ than pre-dose (16.3 and $16.6 \mathrm{bpm})$. A similar effect was also observed in subjects who received concomitant treatment with lesogaberan and esomeprazole: 22.0 and $23.1 \mathrm{bpm}$, respectively, versus 14.6 and $19.0 \mathrm{bpm}$, respectively. However, this effect was not observed in either the lesogaberan alone or concomitant treatment groups at pre-dose on day 7 or at the 3-hour post-dose assessment on day 7 , nor at any timepoint following treatment with esomeprazole alone. A total of seven subjects experienced orthostatic reactions (defined as a drop in diastolic $[>10 \mathrm{mmHg}]$ and/ or a systolic [ $>25 \mathrm{mmHg}$ ] blood pressure at 1 or 3 minutes after moving from a supine to a standing position), mostly on day 7 and with even distribution across treatment periods; only one subject had symptoms (dizziness) at the same time as an orthostatic reaction. Additional orthostatic tests revealed that one subject had

Table II. Geometric mean ratios and 95\% Cls of lesogaberan and esomeprazole area under the plasma concentration-time curve (AUC) during the dosage interval at steady-state $\left(A \cup C_{\tau}\right)$, maximum plasma concentration at steady-state $\left(C_{\max }\right)$, and $A U C$ from time zero until the last quantifiable plasma concentration $\left(A U C_{t}\right)$ when administered concomitantly vs each alone

\begin{tabular}{lll}
\hline Parameter & Geometric mean ratio & $95 \% \mathrm{Cl}$ \\
\hline Lesogaberan & & \\
$\mathrm{AUC}_{\tau}$ & 0.92 & $0.84,1.02$ \\
$\mathrm{C}_{\max }$ & 0.90 & $0.80,1.03$ \\
$\mathrm{AUC}_{\mathrm{t}}$ & 0.92 & $0.84,1.02$ \\
Esomeprazole & & \\
$\mathrm{AUC}_{\tau}$ & 0.97 & $0.89,1.07$ \\
$\mathrm{C}_{\max }$ & 1.04 & $0.93,1.17$ \\
$\mathrm{AUC}_{\mathrm{t}}$ & 0.97 & $0.89,1.07$ \\
\hline
\end{tabular}


Table III. Summary of the secondary pharmacokinetic variables of lesogaberan and esomeprazole when administered alone and concomitantly

\begin{tabular}{llllll}
\hline Parameter & \multicolumn{2}{l}{ Lesogaberan } & & \multicolumn{2}{l}{ Esomeprazole } \\
\cline { 2 - 3 } & alone $(\mathrm{n}=29)$ & + esomeprazole $(\mathrm{n}=28)$ & & alone $(\mathrm{n}=29)$ & + lesogaberan $(\mathrm{n}=28)$ \\
\hline $\mathrm{C}_{\max }[\mu \mathrm{mol} / \mathrm{L}]^{\mathrm{a}}$ & $4.66(22.6)$ & $4.23(38.4)$ & $32.32(32.8)$ & $11.40(34.4)$ & $1.99(25.9)$ \\
$\mathrm{AUC}_{\tau}[\mu \mathrm{mol} . \mathrm{h} / \mathrm{L}]^{\mathrm{a}}$ & $35.00(20.1)$ & $32.33(32.8)$ & $11.25(34.4)$ & $11.21(40.5)$ \\
$\mathrm{AUC}_{\mathrm{t}}[\mu \mathrm{mol} . \mathrm{h} / \mathrm{L}]^{\mathrm{a}}$ & $35.02(20.0)$ & $3.00(2.00-5.00)$ & $2.50(1.00-4.00)$ & $1.51(1.00-4.02)$ \\
$\mathrm{t}_{\max }[\mathrm{h}]^{\mathrm{b}}$ & $3.00(1.60-4.00)$ & $6.59(23.7)$ & $1.42(21.5)$ & $1.40(22.5)$ \\
$\mathrm{t}_{1 / 2}[\mathrm{~h}]^{\mathrm{a}}$ & $6.21(23.5)$ & $32.88(32.9)$ & $10.16(34.4)$ & $10.33(40.5)$ \\
$\mathrm{CL} / \mathrm{F}[\mathrm{L} / \mathrm{h}]^{\mathrm{a}}$ & $30.37(20.1)$ & &
\end{tabular}

a Values are geometric means (coefficient of variation).

b Values are medians (range).

$\mathbf{A} \mathbf{U} \mathbf{C}_{\tau}=$ area under the plasma concentration-time curve during the dosage interval $(\tau)$ at steady-state; $\mathbf{A} \mathbf{U C} \mathbf{C}_{\mathbf{t}}=$ area under the plasma concentration-time curve from time zero until the last quantifiable plasma concentration; $\mathbf{C L} / \mathbf{F}=$ oral clearance at steady-state; $\mathbf{C}_{\max }=$ observed maximum plasma concentration at steady-state; $\mathbf{t}_{1 / 2}=$ terminal elimination half-life; $\mathbf{t}_{\max }=$ time to reach the maximum plasma concentration.

syncope (3 hours post-dose on day 7, esomeprazole alone) and one subject had a vasovagal reaction (same patient as described previously in this section for vasovagal syncope) pre-dose on day 7 , esomeprazole alone. A further two subjects reported dizziness (pre-dose on day 1, lesogaberan alone; and pre-dose on day 2, concomitant treatment with lesogaberan and esomeprazole).

There were no clinically relevant changes in ECG results, laboratory tests, or physical examination findings during the study.

\section{Discussion}

This study investigated the pharmacokinetic interaction between lesogaberan and the PPI esomeprazole in healthy subjects (pharmacokinetic interactions between the two drugs were not expected). Overall, findings were consistent with no relevant pharmacokinetic interaction when the drugs were coadministered at doses of $150 \mathrm{mg}$ (dosing interval 12 hours) and $40 \mathrm{mg}$ once daily. Furthermore, repeated coadministration was well tolerated with no new safety concerns.

The choice of a crossover study design, the selection of the chosen range for the $95 \%$ CI intervals for bioequivalence, and the methods of pharmacokinetic analysis are standard for pharmacokinetic drug-drug interaction studies. It is also standard in drug interaction studies to select high enough doses to ensure that any possible pharmacokinetic interactions are detected. For

Table IV. Number (\%) of subjects with adverse events ${ }^{a}$ during treatment with lesogaberan and esomeprazole, either alone or concomitantly

\begin{tabular}{|c|c|c|c|}
\hline Adverse event & $\begin{array}{l}\text { Lesogaberan } \\
(n=29)\end{array}$ & $\begin{array}{l}\text { Lesogaberan + esomeprazole } \\
(n=29)\end{array}$ & $\begin{array}{l}\text { Esomeprazole } \\
(\mathrm{n}=29)\end{array}$ \\
\hline Paresthesia & $5(17)$ & $4(14)$ & $1(3)$ \\
\hline Pharyngitis & $2(7)$ & $3(10)$ & $0(0)$ \\
\hline Flatulence & $1(3)$ & $4(14)$ & $0(0)$ \\
\hline Diarrhea & $3(10)$ & $1(3)$ & $0(0)$ \\
\hline Rhinitis & $3(10)$ & $1(3)$ & $1(3)$ \\
\hline Dizziness & $1(3)$ & $3(10)$ & $1(3)$ \\
\hline Headache & $1(3)$ & $3(10)$ & $0(0)$ \\
\hline Abdominal pain & $1(3)$ & $2(7)$ & $0(0)$ \\
\hline Nasopharyngitis & $1(3)$ & $2(7)$ & $0(0)$ \\
\hline Myalgia & $0(0)$ & $3(10)$ & $0(0)$ \\
\hline Excoriation & $2(7)$ & $0(0)$ & $0(0)$ \\
\hline
\end{tabular}

a Adverse events experienced by two or more subjects in any treatment group are displayed. 
this reason, the maximal approved dose of esomeprazole $(40 \mathrm{mg})$ was utilized, while the dose of lesogaberan (150 mg; dosing interval 12 hours) was selected because it was in the upper range of the expected therapeutic dose with favorable safety. Although this study could recruit both healthy male and female subjects, the frequency of healthy female subjects meeting the inclusion criterion of being surgically sterilized and aged 18-45 years was low; consequently, only male subjects were recruited. Although there have been slight differences in the pharmacokinetic profile of esomeprazole between male and female subjects (greater systemic exposure in females), these differences have been shown to be statistically insignificant. ${ }^{[15]}$

Comparisons of the $95 \%$ CIs of the geometric mean ratios of systemic exposure $\left(\mathrm{AUC}_{\tau}\right.$ and $\mathrm{C}_{\max }$ ) for lesogaberan and esomeprazole when administered alone and concomitantly were within the standard limits of bioequivalence. Therefore, comparisons indicate that no pharmacokinetic interaction occurs following repeated coadministration of lesogaberan and esomeprazole in healthy subjects.

However, the majority of the study volunteers (>96\%) were Caucasian and, as such, the generalizability of these results to other populations where variable CYP genetic expression is more important is limited. Although pharmacogenetic data were not collected in this study, CYP2C19 polymorphism has been shown to have minimal impact on the pharmacokinetics and pharmacodynamics of esomeprazole in homozygous and heterozygous CYP2C19 extensive metabolizers, ${ }^{[16]}$ and the pharmacokinetic parameters of esomeprazole dosed alone in this study are similar to those previously reported. ${ }^{[15]}$ Furthermore, the ratio and $95 \% \mathrm{CI}$ of $4-\beta \mathrm{OH}$-cholesterol concentrations before and after treatment with lesogaberan indicated that lesogaberan does not induce CYP3A4. This finding is congruent with preclinical studies that show that HepaRG cell CYP3A4, 1A2, 2B6, and 2C9 messenger RNA levels are not increased following cellular incubation with lesogaberan at concentrations of up to $30 \mu \mathrm{mol} / \mathrm{L}^{[14]}$ Furthermore, additional preclinical studies have shown that lesogaberan does not inhibit CYP1A2, 2C9, 2C19, 2D6, or $3 \mathrm{~A} 4{ }^{[17]}$

Although esomeprazole is representative of other PPIs in so far as its metabolism is via CYP3A4 and $2 \mathrm{C} 19$, the fractional dependence on these enzymes for the metabolism of other PPIs and the effects of genetic enzyme variability on the metabolism of other PPIs ${ }^{[16]}$ imply that caution is required when expanding these results to the potential interaction between lesogaberan and other PPIs.

No new safety concerns were raised during this study. The most common adverse events in this study (transient paresthesia, pharyngitis, and flatulence) corresponded to those reported in other clinical studies with lesogaberan. ${ }^{[3,4]}$ Furthermore, the large number of adverse events occurring during treatment with lesogaberan alone or concomitantly with esomeprazole may, in part, be explained by the reporting of paresthesia as multiple events when it occurred at the same time but in different body parts. Although the orthostatic effects that were observed in this study were not of great medical significance in a population of this age, these effects may have a greater impact in elderly patients who may have hypertension.

Although this study was performed using standard methods and analyses, there are some limitations. These include a male and Caucasian study population that limits the generalizability of these findings to the wider public, and the lack of pharmacodynamic data.

\section{Conclusion}

No pharmacokinetic interaction was seen between lesogaberan ( $150 \mathrm{mg}$; dosing interval 12 hours) and esomeprazole ( $40 \mathrm{mg}$ once daily) following repeated coadministration in healthy subjects. Furthermore, repeated coadministration was well tolerated. Therefore, lesogaberan may be used concomitantly in patients receiving esomeprazole, without the risk of pharmacokinetic interaction.

\section{Acknowledgments}

The authors acknowledge Jan Vouis, MD (Principal Investigator) and the research team of Quintiles AB Phase I Unit (Uppsala, Sweden) for conducting the study and the statistical 
analyses; PRA International - Early Development Services (Assen, the Netherlands) for the analyses of biological samples; and Andrew Stead and Simon Lancaster, from inScience Communications, who provided medical writing support funded by AstraZeneca.

The study was funded by AstraZeneca R\&D, Mölndal, Sweden, the manufacturer of esomeprazole and lesogaberan. All authors are present employees of AstraZeneca.

\section{References}

1. Lehmann A, Antonsson M, Holmberg AA, et al. (R)(3-amino-2-fluoropropyl) phosphinic acid (AZD3355), a novel $\mathrm{GABA}_{\mathrm{B}}$ receptor agonist, inhibits transient lower esophageal sphincter relaxation through a peripheral mode of action. J Pharmacol Exp Ther 2009 Nov; 331 (2): 504-12

2. Holloway RH, Dent J. Pathophysiology of gastroesophageal reflux: lower esophageal sphincter dysfunction in gastroesophageal reflux disease. Gastroenterol Clin North Am 1990 Sep; 19 (3): 517-35

3. Boeckxstaens GE, Beaumont H, Mertens V, et al. Effects of lesogaberan, a novel $\mathrm{GABA}_{\mathrm{B}}$-receptor agonist, on reflux and lower esophageal sphincter function in patients with gastroesophageal reflux disease. Gastroenterology 2010; 139 (2): 409-17

4. Boeckxstaens GE, Rydholm H, Lei A, et al. Effect of lesogaberan, a novel $\mathrm{GABA}_{\mathrm{B}}$-receptor agonist, on transient lower esophageal sphincter relaxations in male subjects. Aliment Pharmacol Ther 2010 Mar 4; 31 (11): 1208-17

5. Andersson T, Weidolf L. Stereoselective disposition of proton pump inhibitors. Clin Drug Investig 2008; 28 (5): 263-79

6. Andersson T. Pharmacokinetics, metabolism and interactions of acid pump inhibitors: focus on omeprazole, lansoprazole and pantoprazole. Clin Pharmacokinet 1996 Jul; 31 (1): 9-28

7. Andersson T, Miners JO, Veronese ME, et al. Identification of human liver cytochrome $\mathrm{P} 450$ isoforms mediating omeprazole metabolism. Br J Clin Pharmacol 1993 Dec; 36 (6): 521-30
8. Pichard L, Curi-Pedrosa R, Bonfils C, et al. Oxidative metabolism of lansoprazole by human liver cytochromes $\mathrm{P} 450$. Mol Pharmacol 1995 Feb; 47 (2): 410-8

9. Sohn DR, Kwon JT, Kim HK, et al. Metabolic disposition of lansoprazole in relation to the S-mephenytoin 4'-hydroxylation phenotype status. Clin Pharmacol Ther 1997 May; 61 (5): 574-82

10. Schulz HU, Hartmann M, Steinijans VW, et al. Lack of influence of pantoprazole on the disposition kinetics of theophylline in man. Int J Clin Pharmacol Ther Toxicol 1991 Sep; 29 (9): 369-75

11. Ishizaki T, Horai Y. Review article: cytochrome P450 and the metabolism of proton pump inhibitors - emphasis on rabeprazole. Aliment Pharmacol Ther 1999 Aug; 13 Suppl. 3: 27-36

12. Yasuda S, Horai Y, Tomono Y, et al. Comparison of the kinetic disposition and metabolism of E3810, a new proton pump inhibitor, and omeprazole in relation to S-mephenytoin 4'-hydroxylation status. Clin Pharmacol Ther 1995 Aug; 58 (2): 143-54

13. AstraZeneca. Drug interaction bewteen AZD3355 and nexium [ClinicalTrials.gov identifier NCT00684190]. US National Institutes of health, ClinicalTrials.gov [online]. Available from URL: http://www.clinicaltrials.gov [Accessed 2010 Dec 6]

14. Data on file, AstraZeneca, 2008

15. Andersson T, Hassan-Alin M, Hasselgren G, et al. Pharmacokinetic studies with esomeprazole, the (S)-isomer of omeprazole. Clin Pharmacokinet 2001; 40 (6): 411-26

16. Hunfeld NG, Touw DJ, Mathot RA, et al. A comparison of the acid-inhibitory effects of esomeprazole and pantoprazole in relation to pharmacokinetics and CYP2C19 polymorphism. Aliment Pharmacol Ther 2010 Jan; 31 (1): 150-9

17. Data on file, AstraZeneca, 2001

Correspondence: Mohammad Niazi (MSc, Pharm), Clinical R\&D, AstraZeneca R\&D, Pepparedsleden 1, SE-431 83 Mölndal, Sweden.

E-mail: Mohammad.Niazi@astrazeneca.com 